\title{
DYNAMIC ANALYSIS OF CONSTRAINED MULTIBODY SYSTEMS USING INVERSE KINEMATICS
}

\author{
W. BLAJER, $\dagger$ W. SCHIEHLEN and W. SCHIRM \\ Institute B of Mechanics, University of Stuttgart, Pfaffenwaldring 9, 7000 Stuttgart 80, Germany
}

(Recewed 30 May 1991; received for publication 29 April 1992)

\begin{abstract}
This paper draws attention to the advantages that may arise in the dynamic analysis of constrained multibody systems by applying special algorithms of inverse kinematics developed in the field of robotics. The algorithms result in explicit (recursive) relations for the arbitrary chosen dependent coordinates as functions of the remaining (Independent) ones. Then analogous velocity and acceleration relations are available. Using these explicit closing condition forms, minimal-dimension governing equations of a constrained system can be formulated conveniently. The avoldance of singularities in the analysis is discussed. An illustrative example is included.
\end{abstract}

\section{INTRODUCTION}

During the last two decades a considerable effort has been focused on the development of methods and computational algorithms for mathematical modelling and simulation of multibody systems, see for example Ref. [1]. The methods are mainly concerned with effective formulation and numerical treatment of the dynamic equations of a general multibody system. In a compact form, the equations can be written as

$$
\mathbf{M}(\mathbf{q}, t) \overrightarrow{\mathbf{q}}=\mathbf{h}(\dot{\mathbf{q}}, \mathbf{q}, t),
$$

where $\mathbf{M}$ is an $n \times n$ symmetric positive-definite inertia matrix; $\mathbf{q}$ is an $n$-vector of generalized coordinates; the $n$-vector h represents the applied and gyroscopic forces; $t$ is the time and $n$ is the number of degrees of freedom.

The above formulation is obtained easily for open-loop multibody systems with chain and tree topology. In many technological processes, however, the multibody systems come in contact with the environment and/or closed-loops are designed to improve their mechanical performances. This leads to additional closing conditions (constraints) which have to be included in the dynamical modelling of the problem. The usual approach to the analysis of the constrained/closed-loop multibody system dynamics consists of releasing the system from the additional constraints and/or "cutting" the closed-loops in order to build an "unconstrained" (open-loop) system with the tree structure. The dynamical equations of such a system can then be formulated in the form of equation (1) using the standardized multibody approach. In this case, however, the coordinates $q$ are not independent and, towards retrieving the constrained/closed-loop features of the system, the so-called constraint reactions have to be added to the forces acting on the system. Hence, the dynamical equations of the constrained/closed-loop multibody system transform to

$$
\mathbf{M} \ddot{\mathbf{q}}=\mathbf{h}+\mathbf{C}^{\mathrm{T}} \lambda,
$$

where the term $\mathbf{C}^{\mathrm{T}} \lambda$ expresses the total of the constraint reactions represented in the directions of $\mathbf{q} ; \mathbf{C}$ is an $m \times n$ constraint matrix dependent on $\mathbf{q}$ and $t ; \lambda$ is the $m$-vector of Lagrange undetermined multipliers; and $m$ is the number of constraints on the system. Since $\lambda$ conserves $m$ unknowns, equation (2) must be considered together with the $m$ constraint equations. Limiting ourselves to the position (geometric, holonomic) constraints on the system, their usual analytical form is

$$
\Phi(\mathbf{q}, t)=\mathbf{0},
$$

and the dependence on time may express the prespecified or forced motion, for instance.

tOn leave from the Department of Mechanics, Technical University of Radom, ul. Malczewskiego 29, 26-600 Radom, Poland. 
Equations (2) and (3) form a set of mixed differential and algebraic equations (DAEs). There are at least four leading methods for solving these equations: firstly by direct integration of the DAE system $[2,3]$; secondly by explicit elimination of the multipliers $\lambda[4,5]$; thirdly by using the orthogonal complement matrix or analogous technique [6-8]: fourthly by using the coordinate partitioning $[3,9-11]$. The first three methods treat the constraint equations transformed to the second-order kinematic form, i.e.

$$
\dot{\phi}=\mathbf{C}(\mathbf{q}, t) \ddot{\mathbf{q}}+\mathrm{c}_{0}(\dot{\mathbf{q}}, \mathbf{q}, t)=\mathbf{0},
$$

where $\mathbf{C}=\hat{c} \boldsymbol{\Phi} / \hat{c} \mathbf{q}$ and $\mathbf{c}_{0}=\boldsymbol{C} \dot{\mathbf{q}}+(\hat{c} \boldsymbol{\Phi} / \hat{c} t)$. In consequence, the methods may lead to violation of the constraints defined in equation (3), and special techniques, e.g. Baumgarte's constraint stabilization method $[3,12,13]$, have to be applied to reduce the violation. Moreover, inefficiencies due to the numerical treatment of large-dimension DAE systems, the complex multiplication/ inversion matrix operations leading to the elimination of $\lambda$, and the determination of an orthogonal complement matrix to $\mathbf{C}$, respectively, very often depreciate attractiveness of the methods. The fourth method, which bases on an arbitrary choice of $k=n-m$ independent coordinates $\mathrm{q}_{\text {, from }}$ q. leads to minimal-order governing equations and releases the analysis from the problem of constraint violation. However, while the employed relations between the dependent and independent velocities and accelerations can usually be determined comparatively effectively and automatically by numerical operations, see for example Refs [3,9], the values of dependent coordinates $\mathbf{q}_{D}$ for the current values of $q$, have to be determined by solving the constraint equations in the geometric form of equation (3), and the latter process is often computationally inefficient. The coordinate partitioning formulation may also lead to singularities resulting in the necessity of redefining the set of independent coordinates.

In this paper the attention is drawn on advantages that may arise in the dynamic analysis of constrained multibody systems by applying special algorithms of inverse kinematics developed in the field of robotics: a similar idea was suggested for example in Ref. [14] and exploited in Ref. [4]. Conceptually, the proposed method is equivalent to the coordinate partitioning method mentioned above. As opposed to that formulation, however, the reported approach does not make use of the constraint equations in the form of equation (3), neither their differentiated forms. Instead, by applying procedures of inverse kinematics analysis, recursive relations between the dependent and independent coordinates, velocities and accelerations are introduced explicitly. This enables one to reduce effectively the governing equations to a minimal set and solve them efficiently. Possible variant formulations of the recursive relations for different sets of independent coordinates give a tool for avoiding the encountered singularities by changing the current set of independent coordinates; without any inconsistency in the initial value problem. An example is included to illustrate the advantages of the proposed method.

\section{INVERSE KINEMATICS APPLICATION}

Generally, the inverse kinematics problem of a manipulator is to calculate the joint coordinates and their time derivatives given the prespecified motion of the end-effector with respect to the task reference frame. There are several alternative methods for the determination of manipulator link positions. The most direct method bases on closing the transformation equations (from direct kinematics) formulated, for example, in terms of $4 \times 4$-matrices, for example Refs [15-17]. Conceptually, this approach is equivalent to the formulation of closure condition equations in the form of equation (3). For manipulators with particular geometric properties, closed-form solutions of the closure equations may also be obtained. The other method is based on partitioning a kinematic chain into two open subchains. This approach, used formerly in Ref. [18] for the kinematic analysis of certain spatial mechanisms, has been generalized and formulated systematically in Ref. [19], see also Refs [20-23]. The technique results in selecting relations with the reduced number of unknowns and leads to the recursive analytical solution for the joint coordinates and/or their time derivatives. The inverse kinematics analysis applied in this paper is based mainly on the latter method.

In the considered problem $m$ closing conditions are imposed on an $n$-degree-of-freedom kinematic chain, $m<n$. Hence, $m$ (dependent) joint coordinates $\mathbf{q}_{D}$ can be determined from a set 
of $m$ closure equations which are not, however, introduced here in the implicit form of equation (3). The remaining $k=n-m$ coordinates $\mathbf{q}_{l}$ are independent, and the problem refers to the inverse kinematics analysis of a redundant robot. In order to solve the problem, the independent coordinates have to be conserved, and the bodies connected by these joints can be treated as substitute bodies which geometries depend on the conserved coordinates. Then using the aforementioned procedures for inverse kinematics analysis, due to the conserved coordinates describing the geometries of the substitute bodies, the recursive relations for the dependent coordinates will depend on the independent coordinates. For more details concerning the means by which the recursive relations can be derived, the reader is referred to Ref. [19], and a simple application of the procedures is demonstrated in Section 4 of this paper. Symbolically, the relations can be written as

$$
\mathbf{q}_{D}=\mathbf{g}\left(\mathbf{q}_{t}, t\right),
$$

where $\mathbf{g}$ contains appropriately differentiable functions. Moreover, the inverse kinematics analysis supply the user with analytical recursive formulations for velocities and accelerations, which can be represented in the forms:

$$
\begin{gathered}
\dot{\mathbf{q}}_{D}=\mathbf{A}\left(\mathbf{q}_{l}, t\right) \dot{\mathbf{q}}_{l}+\eta\left(\mathbf{q}_{l}, t\right), \\
\ddot{\mathbf{q}}_{D}=\mathbf{A}\left(\mathbf{q}_{l}, t\right) \ddot{\mathbf{q}}_{l}+\boldsymbol{\xi}\left(\dot{\mathbf{q}}_{l}, \mathbf{q}_{l}, t\right),
\end{gathered}
$$

where the $m \times k$ matrix $\mathbf{A}$ and the $m$-vectors $\boldsymbol{\eta}$ and $\boldsymbol{\xi}$ are the symbolic forms of the actual relations obtained, denoted with the superscript $(\sim)$, i.e. $\mathbf{A}\left(\mathbf{q}_{t}, t\right)=\tilde{\mathbf{A}}(\mathbf{q}, t)=\tilde{\mathbf{A}}\left(\mathbf{g}\left(\mathbf{q}_{l}, t\right)\right)$; $\boldsymbol{\eta}\left(\mathbf{q}_{l}, t\right)=\tilde{\boldsymbol{\eta}}(\mathbf{q}, t)=\tilde{\boldsymbol{\eta}}\left(\mathbf{g}\left(\mathbf{q}_{l}, t\right)\right) ; \quad$ and $\quad \tilde{\xi}\left(\dot{\mathbf{q}}_{l}, \mathbf{q}_{l}, t\right)=\tilde{\xi}\left(\dot{\mathbf{q}}_{l}, \mathbf{q}, t\right)=\tilde{\xi}\left(\dot{\mathbf{q}}_{l}, \mathbf{g}\left(\mathbf{q}_{l}, t\right)\right)$. Conceptually, equations (6) and (7) can be treated as differentiated forms of equation (5), although actually, they are often determined differently in order to achieve the possible simplest forms of equations (6) and (7) $[19,20]$; refer also to the example in Section 4 of this paper.

As shown in the next section, $\mathbf{g}, \mathbf{A}, \boldsymbol{\eta}$ and $\boldsymbol{\xi}$ defined in equations (5)-(7) play a crucial role in the subsequent formulation. As opposed to analogous formulations by application of coordinate partitioning method [3,9] or the method exploited in Ref. [11], the principle benefit of the inverse kinematics application to determine these terms is that all of them are obtained explicitly and analytically, though in a recursive way. Evidently, the choice of a set of independent coordinates $\mathbf{q}$, can be, to some extent, arbitrary. In practice, in order to avoid singularities (matrix A may loose its maximal rank for particular configurations as described in a particular set of $\mathbf{q}_{l}$ ), variant formulations of equations (5)-(7) for different sets of $\mathbf{q}_{\text {, from }} \mathbf{q}$ can be derived and used accordingly. The idea of changing independent coordinates in order to avoid singularities is well known to kinematicians as well, see for example Ref. [24]. As stated in the following, the turn from one to another variant formulation will not result in any inconsistency in the initial value problem. Since equations (5) express, in fact, the constraint conditions on the system, and these equations as well as equations (6) and (7) are used explicitly in the final formulation, the reported analysis is released from the problem of the constraint violation in principle.

In Section 4, equations (5)-(7) are derived for the example case of study. For a general case, the relations may be complex. This fact and the recursive nature of the relations suggest that the proposed approach is rather computer-oriented. On the other hand, the effectiveness in formulating the relations depend greatly on the skill of the investigator in using the inverse kinematics procedures.

\section{MINIMAL-DIMENSION GOVERNING EQUATIONS}

As previously stated, the proposed method is conceptually equivalent to the coordinate partitioning method [9]. Therefore the following sequence will have some resemblence with the formulation of that method proposed in Ref. [11]. Nevertheless, for the sake of completeness and to establish a point of departure for further discussion, some fundamental definitions have to be reviewed from the point of view of the reported method. 
Equation (5) is conceptually equivalent to equation (3) resolved for $\mathbf{q}_{D}$. In other words, equation (5) is another. explicitly resolved form of the constraint equations of the system. Thus, using the following partition of the system's coordinates, see Ref. [25]

$$
\mathbf{q}=\left[\mathbf{q}_{i}^{\top} \mathbf{q}_{D}^{\top}\right]^{\top},
$$

the constraint matrix of $\Phi=-\mathbf{g}\left(\mathbf{q}_{/}, t\right)+\mathbf{q}_{D}=\mathbf{0}$ can be stated as

$$
\hat{C}\left(\mathbf{q}_{/}, t\right)=\left[-\mathbf{A} \mathbf{I}^{(m)}\right] \text {, }
$$

where $\mathrm{I}^{(m)}$ is the $m \times m$ identity matrix; and the superscript $\left(^{\wedge}\right)$ refers to the constraint equations in the resolved form of equation (5). Then, the term $\hat{C}^{\top} \hat{X}$ denotes the total of reaction forces due to $\Phi=0$, represented in the directions of $q$ arranged according to position (8). Obviously, $\bar{C}^{\top} \hat{\lambda}=C^{\top} \lambda$. where $C^{1} \lambda$ is introduced in equation (2), as both $C^{\top} \hat{\lambda}$ and $C^{\top} \lambda$ express the total of constraint-induced forces on the system, and refer only to different possible formulations of equations of these constraints, in the resolved (explicit) form $\boldsymbol{\Phi}=-\mathbf{g}\left(\mathbf{q}_{t}, t\right)+\mathbf{q}_{D}=\mathbf{0}$ followed the application of inverse kinematics algorithms or in the implicit form of equation (3). Using the above definitions. the following kinematic relation can be introduced

$$
\ddot{\mathbf{q}}=\mathbf{D}^{\top} \ddot{\mathbf{q}}_{t}+\left[0^{\top} \boldsymbol{\xi}^{\top}\right]^{\top} \text {. }
$$

where the $k \times n$ matrix $\mathbf{D}$.

$$
\mathbf{D}=\left[\mathbf{I}^{(k)} \mathbf{A}^{\top}\right]
$$

is an orthogonal complement matrix to $\hat{C}$ in the $n$-space, i.e. $\mathbf{D C}^{\top}=\mathbf{0}$, where $\mathbf{0}$ denotes the $k$-dimensional null vector; and $\mathbf{I}^{(k)}$ is the $k \times k$ identity matrix. It is easy to prove that $\mathbf{D}$ is also an orthogonal complement to $C$, i.e. the constraint matrix defined in equation (4). Namely, following the partitioning of equation (8), $\mathbf{C}=\left[\mathbf{C}_{/}^{\top} \mathbf{C}_{D}^{\top}\right]^{\top}$, where $\mathbf{C}_{I}=\partial \Phi / \partial \mathbf{q}_{l}$ and $\mathbf{C}_{D}=\partial \Phi / \partial \mathbf{q}_{D}$, and, comparing $\boldsymbol{\phi}=\mathbf{C}_{l} \dot{\mathbf{q}}_{l}+\mathbf{C}_{D} \dot{\mathbf{q}}_{D}+\hat{c} \boldsymbol{\Phi} \hat{c} t=0$ and $(\boldsymbol{\Phi})^{\circ}=-\mathbf{A} \dot{\mathbf{q}}_{l}+\dot{\mathbf{q}}_{D}-\boldsymbol{\eta}=\mathbf{0}$, it becomes evident that $(\Phi)^{\circ}=\mathbf{0}$ is equivalent to the relation obtained by the left-hand sided premultiplication of $\boldsymbol{\phi}=\mathbf{0}$ by $C_{D}^{-1}$. In other words, $A$ defined in equation (6) and obtained from the inverse kinematics is equivalent to $-C_{D}{ }^{\prime} C_{\text {, }}$ which comes from coordinate partitioning formulation $[3,9,11]$. Hence, $\mathrm{DC}^{\top}=\left[\mathbf{I}^{\mathbf{k}^{k}}\left(-\mathbf{C}_{D}{ }^{1} \mathbf{C}_{l}\right)^{\top}\right]\left[\mathbf{C}_{l}^{\top} \mathbf{C}_{D}^{\top}\right]^{\top}=\mathbf{C}_{I}-\mathbf{C}_{l}\left(\mathbf{C}_{D}^{-1} \mathbf{C}_{D}\right)^{\top}=\mathbf{0}$, i.e. $\mathbf{D}$ is an orthogonal complement matrix both to $\mathcal{C}$ and $\mathrm{C}$. Thus, the superscript $\left({ }^{\wedge}\right)$ has been omitted for $\mathrm{D}$ defined in equation (11).

Projecting equation (2) [or equation (1)] in the directions of $\mathbf{q}_{/}$, i.e. left-hand sided premultiplying the equations by D [6-8], and introducing equations (5), (6) and (11), one obtains the reduceddimension, purely kinetic equations of motion in $\mathbf{q}_{/}$. The equations can be written in the following symbolic form

$$
\mathbf{M}^{\prime}\left(\mathbf{q}_{l}, t\right) \ddot{\mathbf{q}}_{l}=\mathbf{h}^{\prime}\left(\dot{\mathbf{q}}_{l}, \mathbf{q}_{l}, t\right)
$$

where $\mathbf{M}^{\prime}=\mathbf{D M D} \mathbf{D}^{\top}$ and $\mathbf{h}^{\prime}=\mathbf{D}\left(\mathbf{h}-\mathbf{M}\left[\mathbf{0}^{\top} \boldsymbol{\xi}^{\top}\right]^{\top}\right)$. The above equations are equivalent to the formulation obtained in Refs $[9,11]$ by using the coordinate partitioning method. In this case, however, all the terms required for their derivation, that is $\mathbf{A}, \boldsymbol{\xi}, \boldsymbol{\eta}$ and $\mathbf{g}\left(\mathbf{q}_{/}\right)$, are introduced explicitly and analytically by applying the inverse kinematics formulation (note that $\boldsymbol{\eta}$ and $\mathbf{g}\left(\mathbf{q}_{l}\right)$ have to be involved in $\mathbf{q}=\left[\mathbf{q}_{l}^{\top} \mathbf{g}^{\top}\left(\mathbf{q}_{l}, t\right)\right]^{\top}$ and $\dot{\mathbf{q}}=\mathbf{D}^{\top} \dot{\mathbf{q}}_{t}+\left[0^{\top} \boldsymbol{\eta}^{\top}\right]^{\top}$ in order to determine $\mathbf{M}(\mathbf{q}, t)$ and $\mathbf{h}(\dot{\mathbf{q}}, \mathbf{q}, t)$, and then $\mathbf{M}^{\prime}$ and $\mathbf{h}^{\prime}$ ). In practice, it may be convenient to determine $\mathbf{M}^{\prime}$ using the following relation:

$$
\mathbf{M}^{\prime}=\mathbf{M}_{I I}+\left(\mathbf{M}_{I D} \mathbf{A}\right)^{\top}-\mathbf{M}_{I D} \mathbf{A}+\mathbf{A}^{\top} \mathbf{M}_{D D} \mathbf{A},
$$

where $\mathbf{M}_{l l}, \mathbf{M}_{I D}=\mathbf{M}_{D l}^{\top}$, and $\mathbf{M}_{D D}$ are the appropriate block submatrices of $\mathbf{M}$ relating $\mathbf{q}_{l}$ and $\mathbf{q}_{D}$, respectively. Similarly, h' can be conveniently calculated as

$$
\mathbf{h}^{\prime}=\mathbf{h}_{l}+\mathbf{A}^{\top} \mathbf{h}_{D}-\left(\mathbf{M}_{I D}+\mathbf{A}^{\top} \mathbf{M}_{D D}\right) \boldsymbol{\xi} .
$$

Equation (12) represents the dynamic equations transformed to a minimal set, i.e. expressed in $k$ independent coordinates. The computational procedure for numerical treatment of these equations is illustrated in Fig. 1.

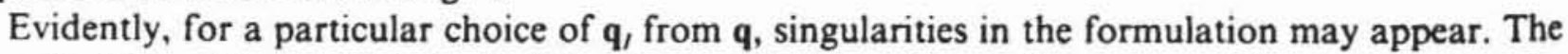
singularities will manifest by indeterminacy of the entries of $\mathbf{A}$ and, with reference to the 


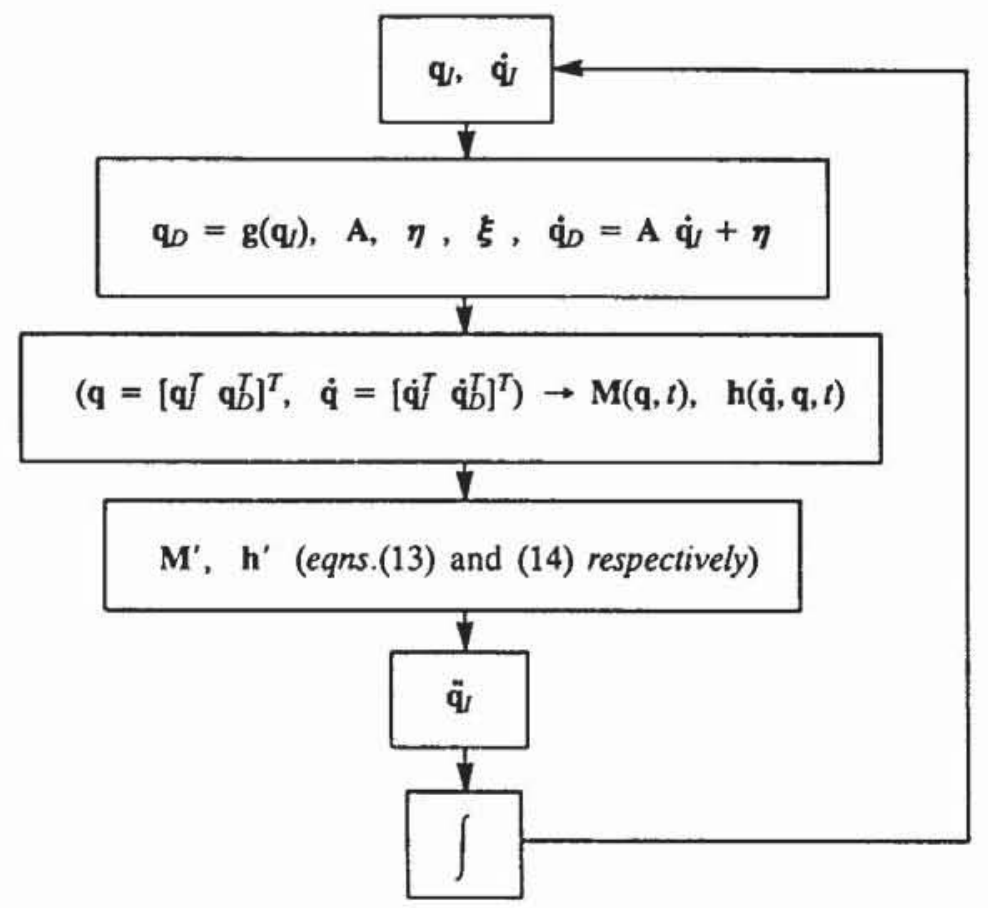

Fig. 1. Computational algorithm for solving equation (12).

formulation $\mathbf{A}=-\mathbf{C}_{D}^{-1} \mathbf{C}_{l}$, correspond to the cases when $\operatorname{det}\left(\mathbf{C}_{D}\right)=0$, see also Refs $[9,11]$. In the case at hand, however, since $\mathbf{A}$ is defined analytically, the singularity points can be predicted in advance [in the standard coordinate partitioning approach, testing $\mathbf{C}_{D}$ for $\operatorname{det}\left(\mathbf{C}_{D}\right) \neq 0$ is suggested]. Then, in order to avoid the singularities in the numerical solution of the problem, the formulation of equations (5)-(7) can be prepared for variant sets of $q_{l}$, and the independent coordinates may be changed accordingly to avoid the singularities in advance. It is worth noting that a transition from one set of independent coordinates to another will require an appropriate rearrangement of position of entries in $\mathbf{M}$ and $\mathbf{h}$ due to the new coordinate partition defined in equation (8). This process, however, can easily be automated. Note also that the change in the independent coordinates will not produce any inconsistency in the initial value problem of the redefined equation (12). As stated above, $\mathbf{q}=\left[\mathbf{q}_{i}^{\mathrm{T}} \mathbf{g}^{\mathrm{T}}\left(\mathbf{q}_{t}, t\right)\right]^{\mathrm{T}}$ and $\dot{\mathbf{q}}=\mathbf{D}^{\mathrm{T}} \dot{\mathbf{q}}_{t}+\left[\mathbf{0}^{\mathrm{T}} \boldsymbol{\eta}^{\mathrm{T}}\right]^{\mathrm{T}}$ are involved to formulate the equations, hence the current values of all entries of $\mathbf{q}$ and $\dot{\mathbf{q}}$ are known at each instant of motion.

Summarizing, the derivation of the minimal-dimension dynamic equations of equation (12) involves the prior formulated dynamic equations for the open-loop/unconstrained system, equation (1), and the explicit formulation of the closing conditions in the form of equations (5)-(7). On this basis, all the subsequent formulation can easily be automated in computations, including eventual changes in the current set of independent coordinates. Moreover, having equations (5)-(7) formulated for different possible sets of closing conditions on the system, constraint addition/deletion problems can be analysed by using the reported approach as well.

\section{EXAMPLE}

Consider a planar four-bar linkage shown in Fig. 2. The linkage can be cut in any of the $O,(i=1, \ldots, 4)$ joints, which will result in a one branch or two branch tree, respectively. Also, the coordinates $\mathbf{q}$ of the unconstrained system may be defined differently. Both these aspects will reflect the formulation of equation (1) and will influence the inverse kinematics algorithms for determining equations (5)-(7). Here, $q=\left[\alpha_{1} \alpha_{2} \alpha_{3}\right]^{\top}$ has been chosen as shown in Fig. 2, and the case may represent a planar manipulator whose end-effector was constrained in the point $\mathrm{O}_{4}$.

Using the approach described in Refs [19-22], the system can be divided into the lower segment, i.e. links 0 (=ground) and 1, and the upper segment, i.e. links 2 and 3. The closure condition for both these segments is

$$
\overline{O_{2} O_{4}}=\overline{O_{1} O_{4}}-\overline{O_{1} O_{2}}=\overline{O_{2} O_{3}}+\overline{O_{3} O_{4}},
$$


where $\left(^{-}\right)$denotes the vector notation. After squaring equation (15), one obtains

$$
l_{0}^{2}+l_{1}^{2}-2 l_{0} l_{1} \cos \left(\pi / 2-\alpha_{1}\right)=l_{2}^{2}+l_{3}^{2}+2 l_{2} l_{3} \cos \alpha_{3},
$$

where $l_{0}, l_{1}, l_{2}$ and $l_{3}$ are described in Fig. 2.

Due to the segmentation of the linkage through the joints $O_{2}$ and $O_{4}$, the coordinate $\alpha_{2}$, which relates the joint $O_{2}$, is not involved in equation (16). Hence, either $\alpha_{1}$ or $\alpha_{3}$ can be chosen as an independent coordinate of the system. From equation (16) one obtains

$$
\begin{aligned}
& x_{1}= \pm \arccos \left(\frac{l_{0}^{2}+l_{1}^{2}-l_{2}^{2}-l_{3}^{2}-2 l_{0} l_{1} \sin \alpha_{1}}{2 l_{2} l_{3}}\right), \\
& x_{1}=\frac{\pi}{2} \pm \arccos \left(\frac{l_{0}^{2}+l_{1}^{2}-l_{2}^{2}-l_{3}^{2}-2 l_{2} l_{3} \cos \alpha_{3}}{2 l_{0} l_{1}}\right) .
\end{aligned}
$$

In the following, cases $a$ and $b$ will relate to the choice of $x_{1}$ or $x_{3}$ as an independent coordinate, respectively. Note that for a given independent coordinate, two possible solutions for the dependent coordinate exist. Then, the complementary relation for determination of $x_{2}$ can be obtained from the vector projections on the direction of $\mathrm{O}_{2} \mathrm{O}_{3}$ and its perpendicular, i.e.

$$
\begin{aligned}
& \sin x_{2}=\frac{l_{0} l_{2} \cos x_{1}+l_{0} l_{3} \cos \left(x_{1}+x_{3}\right)+l_{1} l_{3} \sin x_{3}}{l_{0}^{2}+l_{1}^{2}-2 l_{0} l_{1} \sin x_{1}}, \\
& \cos x_{2}=\frac{l_{0} l_{2} \sin x_{1}+l_{0} l_{3} \sin \left(x_{1}+x_{3}\right)-l_{1} l_{3} \cos x_{3}-l_{1} l_{2}}{l_{0}^{2}+l_{1}^{2}-2 l_{0} l_{1} \sin x_{1}} .
\end{aligned}
$$

Equations (18) determine explicitly $x_{2}$ as a function of $x_{1}$ and $x_{3}$ and, after introducing equations (17), express recursively $x_{2}\left(x_{1}\right)$ or $x_{2}\left(x_{3}\right)$, respectively for case $a$ or $b$.

The differentiation of equations (17) leads to:

$$
\begin{aligned}
& \dot{x}_{3}=\frac{l_{0} l_{1}}{l_{2} l_{3}} \frac{\cos x_{1}}{\sin x_{3}} \dot{x}_{1}, \\
& \dot{x}_{1}=\frac{l_{2} l_{3}}{l_{0} l_{1}} \frac{\cos x_{3}}{\cos x_{1}} \dot{x}_{3} .
\end{aligned}
$$

Then, from the velocity projections, one can receive

$$
\dot{x}_{2}=-\frac{l_{0}}{l_{3}} \frac{\cos \left(x_{1}+x_{2}\right)}{\sin \alpha_{3}} \dot{x}_{1}-\dot{x}_{3} .
$$

Now, introducing equations (19), $\dot{\alpha}_{2}$ can be stated as a linear function of the independent velocities $\dot{\alpha}_{1}$ or $\dot{x}_{3}$, respectively, i.e.

$$
\begin{aligned}
& \dot{\alpha}_{2}=-\frac{l_{0} l_{1} \cos \alpha_{1}+l_{0} l_{2} \cos \left(\alpha_{1}+\alpha_{2}\right)}{l_{2} l_{3} \sin \alpha_{3}} \dot{\alpha}_{1}, \\
& \dot{\alpha}_{2}=-\left(\frac{l_{2} \cos \left(\alpha_{1}+\alpha_{2}\right)}{l_{1} \cos \alpha_{1}}+1\right) \dot{\alpha}_{3} .
\end{aligned}
$$

Using equations (19) and (21), the matrix $\mathbf{D}$ defined in equation (11) can be stated as follows:

$$
\begin{aligned}
& \mathbf{D}_{a}=\left[\begin{array}{lll}
1 & -\frac{l_{0} l_{1} \cos \alpha_{1}+l_{0} l_{2} \cos \left(\alpha_{1}+\alpha_{2}\right)}{l_{2} l_{3} \sin \alpha_{3}} & \frac{l_{0} l_{1}}{l_{2} l_{3}} \cdot \frac{\cos \alpha_{1}}{\sin \alpha_{3}}
\end{array}\right], \\
& \mathbf{D}_{b}=\left[\begin{array}{lll}
1 & \frac{l_{2} l_{3}}{l_{0} l_{1}} \cdot \frac{\sin \alpha_{3}}{\cos \alpha_{1}}-\left(\frac{l_{2} \cos \left(\alpha_{1}+\alpha_{2}\right)}{l_{1} \cos \alpha_{1}}+1\right)
\end{array}\right],
\end{aligned}
$$




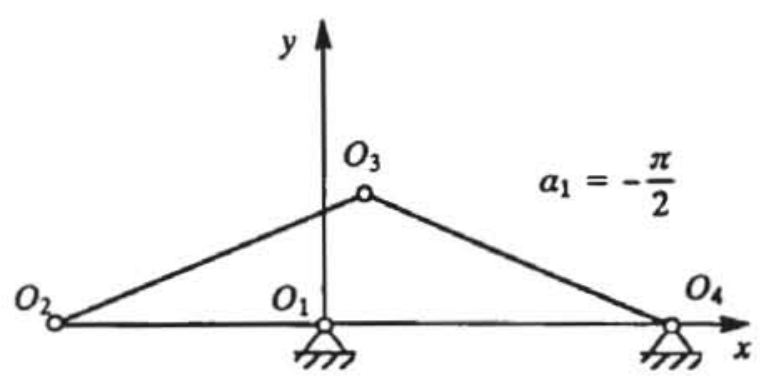

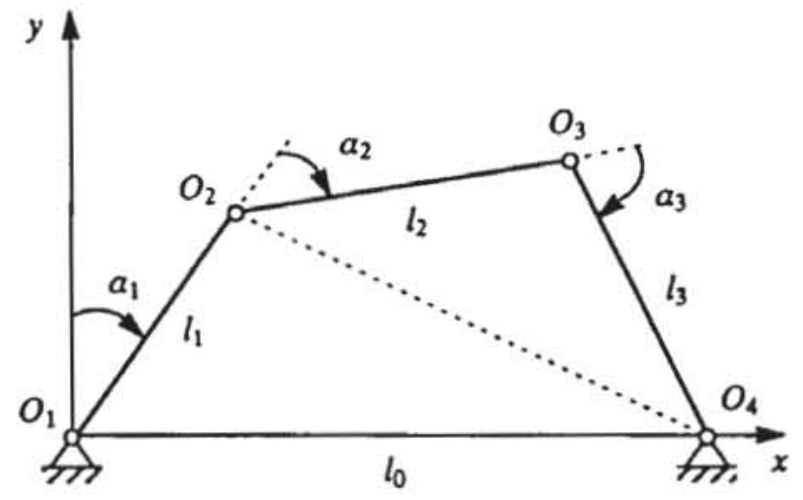

Fig. 2. A four-bar mechanism.

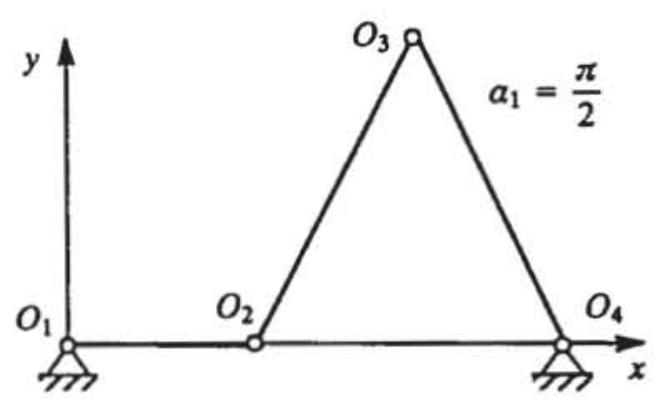

Fig. 3. Singular configurations.

respectively for $\mathbf{q}_{a}=\left[\alpha_{1} \alpha_{2} \alpha_{3}\right]^{\top}$ and $\mathbf{q}_{l a}=\left[\alpha_{1}\right]$; and $\mathbf{q}_{b}=\left[\alpha_{3} \alpha_{1} \alpha_{2}\right]^{\top}$ and $\mathbf{q}_{l b}=\left[\alpha_{3}\right]$. Then, the vector $\xi$ introduced in equation (7) can be obtained after differentiating equations (19) and (21). Respectively,

$$
\begin{aligned}
& \xi_{a}=\dot{\alpha}_{1}\left[\frac{K_{1}+K_{2}}{L_{1}}-\frac{K_{1}}{L_{1}}\right]^{\mathrm{T}}, \\
& \xi_{b}=\dot{\alpha}_{3}\left[\frac{K_{3}}{L_{2}} \frac{K_{4}}{L_{2}}\right]^{\mathrm{T}},
\end{aligned}
$$

where

$$
\begin{aligned}
& K_{1}=l_{0} l_{1}\left(\dot{\alpha}_{1} \sin \alpha_{1} \sin \alpha_{3}+\dot{\alpha}_{3} \cos \alpha_{1} \cos \alpha_{3}\right), \\
& K_{2}=l_{0} l_{2}\left[\left(\dot{\alpha}_{1}+\dot{\alpha}_{2}\right) \sin \left(\alpha_{1}+\alpha_{2}\right) \sin \alpha_{3}+\dot{\alpha}_{3} \cos \left(\alpha_{1}+\alpha_{2}\right) \cos \alpha_{3}\right], \\
& L_{1}=l_{2} l_{3} \sin ^{2} \alpha_{3}, \\
& K_{3}=l_{2} l_{3}\left(\dot{\alpha}_{3} \cos \alpha_{3} \cos \alpha_{1}+\dot{\alpha}_{1} \sin \alpha_{3} \sin \alpha_{1}\right), \\
& K_{4}=l_{0} l_{2}\left[\dot{\alpha}_{1} \sin \alpha_{2}+\dot{\alpha}_{2} \sin \left(\alpha_{1}+\alpha_{2}\right) \cos \alpha_{1}\right], \\
& L_{2}=l_{0} l_{1} \cos ^{2} \alpha_{1} .
\end{aligned}
$$

It is evident that, after introducing equations (17)-(19) and (21), the matrix $\mathbf{D}$ and the vector $\xi$ defined in equations (22) and (23), respectively, can be symbolically written as $\mathbf{D}_{a}=\mathbf{D}_{a}\left(\alpha_{1}\right)$ and $\mathbf{D}_{b}=\mathbf{D}_{b}\left(\alpha_{3}\right)$, and $\boldsymbol{\xi}_{a}=\xi_{a}\left(\dot{\alpha}_{1}, \alpha_{1}\right)$ and $\boldsymbol{\xi}_{b}=\boldsymbol{\xi}_{b}\left(\dot{\alpha}_{3}, \alpha_{3}\right)$, respectively.

Given the dynamical model of the open-loop system (cut in the point $O_{4}$, see Fig. 2), i.e. given $\mathbf{M}$ and $\mathbf{h}$ defined in equation (1), g, D, and $\boldsymbol{\xi}$ introduced in equations (17), (18), (22) and (23), respectively, suffice to derive the minimal-dimension dynamic equations in the form of equation (12) [for the case at hand, $\boldsymbol{\eta}=\mathbf{0}$ since equations (17) and (18), referring to equation (5), do not depend explicitly on time]. In this example, one dynamical equation will be obtained, $k=1$. Due to the analytical complexity of the equation, it will not be written in this paper.

The above formulation allows one to introduce either $\alpha_{1}$ or $\alpha_{3}$ as an independent coordinate. In each case, singularities in the respective formulation exist: for case $a, \mathbf{q}_{l a}=\left[\alpha_{1}\right]$, the singularity point 
is achieved when $\sin \alpha_{3}=0$; and for case $b, q_{l b}=\left[\alpha_{3}\right]$, when $\cos \alpha_{1}=0$. At these points, the entries of respectively formulated $\mathbf{A}$ become indeterminate, which results in the indeterminancy of $\mathbf{D}$ and $\boldsymbol{\xi}=\dot{A} \dot{q}_{l}$. However, excluding the cases when $l_{0}=\mp l_{1} \mp l_{2} \mp l_{3}$, the singularity points for cases $a$ and $b$ will never come together. Thus, as mentioned in Section 3, when approaching a particular singularity point in the process of numerical simulation, the current independent coordinate can be changed to the other one. This can be done due to the reported variant formulations, cases $a$ and $b$, of the inverse kinematics formulas.

It may be of worth to discuss the meaning of the encountered singularities in more detail. Let us analyse, for example, the singularities for case $b, \mathbf{q}_{1 b}=\left[x_{3}\right]$. Four singular configurations of the linkage are possible; two of them are shown in Fig. 3, and the remaining two singular configurations are their mirror pictures with respect to the $x$-axis. Note that at these configurations, the distance $\left|\bar{O}, O_{4}\right|$. used to define the closure condition of equation (15), achieves extrema, or due to the formulation of equation (16), $\cos \alpha_{3}$ achieves extrema. In other words, from the extremum condition which results in $\dot{x}_{3} \sin \alpha_{3}=0$, it follows that $\dot{\alpha}_{3}=0$ at these points, since $\sin x_{3} \neq 0$. Thus, at these configurations, $\dot{x}_{3}$ cannot serve as an independent velocity to determine the dependent ones, i.e. $\dot{x}_{1}$ and $\dot{x}_{2}$, according to equations (19b) and (21b), respectively, or according to equation (6) in general. Moreover, since the dependent velocities $\dot{\mathbf{q}}_{b}$ do not equal zero (in general) at these configurations, the indeterminancy of entries of $\mathbf{A}$ becomes evident as resulting in $\mathbf{A} \dot{q}_{t}={ }^{\prime} x \cdot 0$ " in equation (6). Concluding, the singularities for a particular choice of $\mathbf{q}$, from $\mathbf{q}$ are of kinematical nature. i.e. are due to the particular choice of $\mathbf{q}_{\boldsymbol{l}}$ from $q$.

\section{DISCUSSION AND CONCLUSIONS}

Given the inverse kinematics formulations of equations (5)-(7), the reported approach seems to be extremely efficient and attractive in applications. Simple and evident manipulations leading to the dynamical equations in independent coordinates can easily be automated in computations, and the analysis is released from the problem of constraint violation. Automatical avoidance of singularities in the analysis can also be achieved. Moreover, the approach is suited for analysing the constraint addition/deletion problems as well.

A possible disadvantage of the proposed method, as compared with the other methods for dynamic analysis of constrained multibody systems, may be a considerable amount of labour devoted usually to derive equations (5)-(7), and very often, complexity of these (recursive) equations. As a rule, the closing conditions in the common form of equation (3), i.e. $\boldsymbol{\Phi}(\mathbf{q}, t)=\mathbf{0}$ are simpler to introduce; though their differentiated forms $\phi=0$ and $\phi=0$, used in the other methods, involve often some complexity and difficulty in derivation. On the other hand, the efficiency of the inverse kinematics formulations depend greatly on the skill of the user, and the conjecture of the authors is that, for many practical applications, the inverse kinematics formulation of the closing conditions may be even superior to the traditional formulation.

The proposed method is rather computer-oriented. Given $\mathbf{M}, \mathbf{h}, \mathbf{g}, \mathbf{A}, \boldsymbol{\eta}$ and $\boldsymbol{\xi}$, the entries of $\mathbf{M}^{\prime}$ and $h^{\prime}$ defined in equation (12) can easily be obtained by standardized numerical matrix multiplication operations; the analytical derivation of the corresponding relations may be a laborious and complicated task. Moreover, many methods for obtaining equations of motion of open-loop systems [1] result in $\mathbf{M}$ and $\mathbf{h}$ determined numerically. Also, the recursive nature of the symbolically written relation of equation (6) may impede the analytical derivation of $\mathbf{A}$ [see the transformation from equation (20) to (21) in the reported example; in general, such a transformation may be not so evident]. On the other hand, a symbolical multibody system formalism like NEWEUL $[1,26]$ can be applied to derive equation (1) and the required manipulations symbolically. Using this formalism, given $\mathbf{q}_{D}=\mathbf{g}\left(\mathbf{q}_{l}, t\right)$, the entries of $\mathbf{A}, \boldsymbol{\eta}$ and $\boldsymbol{\xi}$ can be derived symbolically as well. As a result, equation (12) can be obtained semi-automatically in the symbolic form, though the final form of these equations may be complex. These topics are not the scope of this paper, however. 


\section{REFERENCES}

1. W. Schiehlen, Multibody Systems Handbook. Spnnger, Berlin (1990).

2. E. J. Haug. S. C. Wu and S. M. Yang, Mech. Mach. Theory 21, 401 (1986).

3. P. E. Nikravesh, Computer Aided Analysis and Optimization of Mechanical System Dynamics (Ed. E. J. Haug), NATO ASI Series, Vol. F9, pp. 351-368. Springer, Berlin (1984).

4. W. Schiehlen, Computer Meth. Appl. Mech. Engng 90, 569 (1991).

5. J. Wittenburg, Dynamics of Systems of Rigid Bodies, p. 176. Teubner, Stuttgart (1977).

6. W. Blajer, Mech. Res. Commun. 18, 293 (1991).

7. H. Hemami and F. C. Weimer, Trans. ASME Jl Appl. Mech. 48, 177 (1981).

8. J. W. Kamman and R. L. Huston. Trans. ASME Jl Appl. Mech. 51, 899 (1984).

9. R. A. Wehage and E. J. Haug. Trans. ASME Jl Mech. Des. 104, 247 (1982).

10. P. E. Nikravesh, J Non-Linear Mech. 25, 143 (1990).

11. C. Wampler. K. Buffinton and J. Shu-hui, Trans. ASME Jl Appl. Mech. 52, 465 (1985).

12. J. Baumbarte, Computer Meth. Appl. Mech. Engng 1, 1 (1972).

13. G.-P. Ostermeyer, Real-tume Integration Methods for Mechantcal System Simulation (Eds E. J. Haug and R. C. Deyo), NATO ASI Senes, Vol. F69, pp. 193-207. Springer, Berlın (1990).

14. A. A. Shabana. Dynamics of Multubody Systems, p. 406. John Wiley, New York (1989).

15. R. P. Paul, Robot Manipulators: Mathematics, Programming and Control. MIT Press, Cambridge, MA (1981).

16. F. L. Litvin and V. Parentı Castelli, Trans. ASME Jl Mech. Transm. Automn Des. 107,170 (1985).

17. F L. Litvin, V. Parentı Castelı and R. H. Phillıps, Mech. Mach. Theory 21, 173 (1985).

18. F. L Litvin, Mech. Mach. Theory 10, 315 (1975).

19. C. Woernle, Ein systematisches Verfahren zur Aufstellung der geometrischen Schließbedingungen in kinematischen Schleifen mut Anwendung bet der Rückwärtstransformation für Industrieroboter. VDI, Düsseldorf (1988).

20. M. Hiller and A. Kecskemethy, Proc. 7th Wld Congr. Theory of Machines and Mechanism, Sevilla, Spain, p. 425 (1987).

21. M. Hiller and C. Woernle, Proc. 7th Wld Congr. Theory of Machines and Mechanism, Sevilla, Spain, p. 1135 (1987).

22 M. Hiller and C. Woernle, Proc. IEEE Int. Conf. Robots and Automation, Philadelphia, p. 846 (1988).

23. M. Eppinger and E. Kreuzer, Proc, VIIIth CISM-IFToMM Symp., RoManSy'90, Cracow, Poland, p. 53 (1990).

24. M. S. C. Yuan, F. Freudensteın and L. S. Woo, Trans. ASME Jl Engng Ind., p. 67 (1971).

25. W. Schiehlen, Report DLR No. DL/90/WOS/1 Department of Mechanical Engineering, Queen's University, Kingston (1990).

26. E. Kreuzer and G. Leister, Report Anleitung AN-23, Instıtute B of Mechanics, University of Stuttgart (1991).

\section{DYNAMIKANALYSE VON MEHRKÖRPERSYSTEMEN MIT GESCHLOSSENEN KINEMATISCHEN SCHLEIFEN MIT HILFE DER INVERSEN KINEMATIK}

Zusammenfassung - Be1 der Entwicklung von Algorithmen zur Modellierung und Simulation von Mehrkörpersystemen ist die effiziente Formulierung und numerısche Behandlung der Bewegungsgleichungen eines allgemeınen Mehrkörpersystems von großer Bedeutung.

Diese Arbeit behandelt Mehrkörpersysteme mit geschlossenen kinematischen Schleifen. Für derartige Mehrkörpersysteme können durch die Anwendung spezieller Algorithmen der inversen Kinematik, welche sich auf dem Gebiet der Roboterdynamik bewährt haben, Vorteile bei der dynamischen Analyse entstehen. Die Idee dieses Verfahrens liegt in der expliziten Beschreibung rekursiver Beziehungen zwischen abhängigen und unabhängigen Koordinaten, Geschwindigkeiten und Beschleunigungen. Bei Anwendung dieser Bezıehungen auf die Bewegungsgleichungen eines Mehrkörpersystems mit geschlossenen kinematischen Schleifen in der Form

$$
\mathbf{M} \ddot{q}=h+C^{\top} \lambda,
$$

werden diese auf eine Mınimalform reduziert, d.h.

$$
\mathbf{M}^{\prime}\left(\mathbf{q}_{l}, t\right) \mathbf{q}_{t}=\mathbf{h}^{\prime}\left(\dot{\mathbf{q}}_{t}, \mathbf{q}_{l}, t\right),
$$

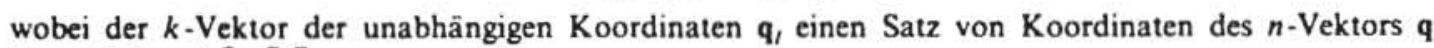
darstellt $\left(\mathbf{q}=\left[\begin{array}{l}\mathbf{q}_{i}^{\top} \\ \mathbf{q}_{b}^{\top}\end{array}\right]^{\top}\right)$.

Durch einen erhōhten Aufwand beı der Gleichungsgenerıerung gewinnt man die minimale Anzahl von Gleichungen, welche sich numerisch als vorteilhaft erwiesen haben. Allerdings können bei dieser Beschreibung Singularitāten im Laufe der Analyse auftreten, welche vermieden werden müssen.

Am Beispiel eines ebenen Viergelenkes wird der vorgeschlagene Algorithmus aufgezeigt und das Ergebnis diskutiert. 